Cahiers $d u$ MONDE RUSSE

\section{Cahiers du monde russe}

Russie - Empire russe - Union soviétique et États indépendants

48/2-3 | 2007

Les résonances de 1905

\title{
Les socialistes français et la révolution de 1905
}

\author{
Gilles Candar
}

\section{OpenEdition \\ Journals}

Édition électronique

URL : https://journals.openedition.org/monderusse/9011

DOI : 10.4000/monderusse. 9011

ISSN : $1777-5388$

Éditeur

Éditions de l'EHESS

Édition imprimée

Date de publication : 15 avril 2007

Pagination : $365-378$

ISBN : 978-2-7132-2147-7

ISSN : $1252-6576$

Référence électronique

Gilles Candar, "Les socialistes français et la révolution de 1905 », Cahiers du monde russe [En ligne], 48/2-3 | 2007, mis en ligne le 01 janvier 2007, consulté le 04 septembre 2022. URL : http://

journals.openedition.org/monderusse/9011; DOI : https://doi.org/10.4000/monderusse. 9011

Tous droits réservés 
chercher : repérer : avancer

Cet article est disponible en ligne à l'adresse :

http://www.cairn.info/article.php?ID REVUE=CMR\&ID NUMPUBLIE=CMR 482\&ID ARTICLE=CMR 4820365

Les socialistes français et la révolution de 1905

\author{
par Gilles CANDAR
}

\title{
Editions de l'EHESS | Cahiers du monde russe
}

\author{
$2007 / 2-3-$ Vol 48 \\ ISSN 1252-6576 | ISBN 9782713221477 | pages 365 à 378
}

Pour citer cet article :

_Candar G., Les socialistes français et la révolution de 1905, Cahiers du monde russe 2007/ 2-3, Vol 48, p. 365-378.

Distribution électronique Cairn pour les Editions de l'EHESS.

(C) Editions de l'EHESS. Tous droits réservés pour tous pays.

La reproduction ou représentation de cet article, notamment par photocopie, n'est autorisée que dans les limites des conditions générales d'utilisation du site ou, le cas échéant, des conditions générales de la licence souscrite par votre établissement. Toute autre reproduction ou représentation, en tout ou partie, sous quelque forme et de quelque manière que ce soit, est interdite sauf accord préalable et écrit de l'éditeur, en dehors des cas prévus par la législation en vigueur en France. Il est précisé que son stockage dans une base de données est également interdit. 


\section{LES SOCIALISTES FRANÇAIS ET LA RÉVOLUTION DE 1905}

Les socialistes français se sont-ils intéressés à la révolution russe de 1905 ? Et si oui, comme cela fut probablement en partie le cas, selon quelles modalités et dans quelle mesure? Leurs analyses furent-elles absolument originales ou rencontrèrent-elles celles d'autres courants politiques ? Étaient-ils d'accord entre eux ou cet événement fut-il sujet de dissensions ? Cette révolution les a-t-elle informés ou influencés? Toutes ces questions, assurément peu débattues aujourd'hui, rappellent d'anciennes discussions, qui eurent leur moment de notoriété, sur les rapports entre la France et la Russie, la nature du socialisme ou du communisme, en France et ailleurs. Elles offrent aussi la possibilité de poursuivre la réflexion sur les relations réciproques entre histoires nationales.

Russes et Français ont souvent pensé que la dernière question appelait une réponse positive. Ainsi, l'historien soviétique Albert Manfred considérait que la révolution de 1905 avait marqué une césure dans l'histoire du mouvement ouvrier français ${ }^{1}$. La même thèse se retrouvait sans trop de surprise dans des articles publiés par des responsables érudits du parti communiste français, dans le cadre de ce que nous pourrions appeler une histoire militante (Georges Cogniot, Jean Bruhat, Jacques Duclos $)^{2}$. Sans appartenir à cette mouvance idéologique, le grand historien Jacques Droz, dont les analyses se déployaient dans un tout autre registre, estimait lui aussi qu'elle avait eu une influence forte, en ceci qu'elle avait réveillé le souvenir de journées révolutionnaires, popularisé la notion de grève générale et encouragé les minorités d'extrême gauche dans le syndicalisme et le socialisme

1. Cf. Albert Manfred, « La révolution russe de 1905/1907 et le mouvement révolutionnaire en France », Recherches soviétiques, 5, septembre-octobre 1956, repris et complété dans Essais sur l'histoire de France du XVIII s. au XX $X^{e}$ s., M. : Éd. du Progrès, 1969, 623 p.

2. Cf. notamment Georges Cogniot, « 1905 : la première révolution russe et le mouvement ouvrier français », Les Cahiers du Communisme, mars 1975 et Jean Bruhat, « Le Dimanche rouge de Pétersbourg », L'Humanité, 14 janvier 1975. 
français ${ }^{3}$. Aujourd'hui, ce dossier peut être repris en étant moins dominé par le poids de 1917 et celui de toute l'histoire de l'Union soviétique et du mouvement communiste qui pesèrent longtemps sur 1905. De fait, le débat historien doit chercher à répondre à trois questions aussi simples que déterminantes.

\section{Les socialistes français se sont-ils intéressés à la révolution russe de 1905 ?}

À cette première question, nous répondons sans hésitation par l'affirmative, ne serait-ce que parce que les affaires russes intéressaient au premier chef les Français. Chacun sait, et chacun savait à l'époque, que la Russie était la grande nation amie et alliée qui avait permis à la France de sortir de son isolement après la défaite de 1871. Massivement, les Français étaient satisfaits de cette alliance, gage de paix d'ailleurs plus qu'atout pour une revanche qui, en 1904-1905, n'apparaissait guère d'actualité. En outre, la Russie était un pays où l'épargne française aimait à investir. Quelques ombres cependant ternissaient ce tableau : à gauche, donc surtout chez les socialistes, mais pas seulement, on ne pouvait oublier tout à fait que la Russie était la clef de voûte d'une Europe monarchique, que la Russie était le pays de la réaction par excellence. Hugo l'avait exprimé avec une ample et efficace solennité dans Châtiments :
Peuple russe, tremblant et morne, tu chemines,
Serf à Saint-Pétersbourg, ou forçat dans les mines.
Le pôle est pour ton maître un cachot vaste et noir ;
Russie et Sibérie, ô czar ! Tyran, vampire !
Ce sont les deux moitiés de ton funèbre Empire ;
L'une est l'Oppression, l'autre est le Désespoir.

Cependant, ce sentiment supposait déjà une certaine culture, une formulation politique, et se manifestait moins ouvertement depuis que les deux pays étaient alliés. Après tout, même un jeune député républicain avancé comme Jean Jaurès s'était montré favorable à cette alliance lors de sa préparation. Dès lors, grande nation alliée, la Russie était devenue pour les Français, du moins pour leur presse, pour l'opinion éclairée en quelque sorte - sans doute pas dans tous les terroirs ni tous les faubourgs - , un centre d'intérêt puissant, une garantie de développement et l'objet de nombreuses sympathies.

Les socialistes, courant politique encore neuf et assez marginal au début du siècle, avaient conservé du vieux legs républicain une hostilité déclarée au tsarisme. Ils y étaient encouragés par les nombreux réfugiés qui vivaient et militaient en France : ne serait-ce que sur ce sujet, il conviendrait de citer Lavrov décédé en 1900, il était resté une référence - , Rappoport, Trockij, Lenin, Martov,

3. Cf. Jacques Droz, Introduction à l'Histoire générale du socialisme, tome I, P. : PUF, 1974. J'ai plaisir à saluer ici avec respect et reconnaissance la mémoire de ce professeur exceptionnel et de cet historien aussi subtil qu'érudit, qui dirigea mon premier travail sur ce sujet, « La presse socialiste parisienne et la révolution russe de 1905 », mémoire de maîtrise, Paris I, 1975. 
Steklov, Rubanovič, également Danevič et Leiteisen, moins connus mais actifs en 1905... Ainsi, les socialistes n'avaient pas pris position pour la Russie lors de la guerre russo-japonaise. Bien plus, à tort ou à raison, cette guerre avait semblé pour beaucoup annoncer des changements révolutionnaires, fondés sur des souvenirs historiques largement partagés au sein du monde militant. Ceux qui se situaient le plus à gauche, ou les plus doctrinaires si l'on préfère, parlaient de « guerre sainte ${ }^{4}$ comme Charles Bonnier, intellectuel influent chez les guesdistes, ou de «guerre féconde $»^{5}$ tel le « vaillantiste » Louis Dubreuilh. Ils espéraient que la guerre allait amener la chute du tsarisme de la même manière qu'elle avait entraîné en France la fin de Louis XVI ou de Napoléon III. Cette analyse n'était pas toutefois celle de tous les socialistes. Les plus modérés étaient sans doute plus pacifiques, non seulement par tempérament, mais avant tout parce que depuis l'affaire Dreyfus, Jaurès et ses amis souhaitaient lier profondément l'avenir socialiste à une revendication d'idéal, à une exigence d'éthique portée par l'ensemble de l'humanité. La paix devenait une valeur en soi, et pas seulement un bénéfice secondaire de l'émancipation du prolétariat. Jaurès s'en prenait aux «théoriciens de la guerre, plagiaires attardés de Proudhon » et estimait que non seulement la guerre n'était pas nécessaire à la révolution, mais qu'aujourd'hui, sa cause « était liée à celle de la paix » ${ }^{6}$. En outre, sans doute davantage que beaucoup d'autres, il ressentait les possibilités de régression et toutes les conséquences, humaines, politiques et sociales d'un conflit armé généralisé. Il les exprima avec une certaine force dans divers articles et il convient de relever que ce fut précisément la guerre russo-japonaise qui marqua en lui la montée de l'inquiétude devant les risques de guerre mondiale: son discours de Saint-Étienne (13 février 1904) fut des plus explicites à ce sujet et marqua sans doute la césure la plus importante en politique extérieure chez lui ${ }^{7}$. Remarquons aussi, que sur ce sujet comme sur bien d'autres, Jean Allemane, peu modéré de tempérament, ancien communard et typographe, prototype d'un socialisme parisien assez gouailleur, anticlérical et antimilitariste, partageait avec Jaurès le même parti pris de principe en faveur de la paix. De plus, les socialistes les plus favorables à la paix étaient souvent les plus engagés en faveur de l'alliance avec la Grande-Bretagne et évitaient pour le moins de soutenir une Russie jugée expansionniste et impérialiste.

Quel que soit donc le regard historiographique actuel sur les liens entre guerre russo-japonaise et révolution ${ }^{8}$, toujours discutables bien entendu, il est certain qu'à l'époque, des troubles, des révoltes, sinon une révolution étaient attendus, et un

4. Charles Bonnier, « Le péril cosaque », Le Socialiste, 7 février 1904.

5. Titre de son article dans Le Socialiste, 25 décembre 1904.

6. Jean Jaurès, « Guerre et paix », L'Humanité, 24 novembre 1904.

7. En attendant la publication du volume correspondant des Euvres de Jean Jaurès chez Fayard, se reporter à La Revue socialiste d'avril 1904 ou au volume La Paix menacée, éd. Max Bonnafous, P. : Rieder, 1931, p. 63-90. Sur ce tournant, cf. « 1905 et le nationalisme. Y a-t-il des tournants historiques ? », Mil Neuf Cent, revue d'histoire intellectuelle, 19, 2001.

8. Cf . François-Xavier Coquin, 1905, la révolution russe manquée, Bruxelles : Complexe, 1985 . 
oracle de politique extérieure comme Francis de Pressensé pouvait évoquer sans ridicule la venue d'une « aube de révolution » ${ }^{9}$. Le Dimanche rouge du 22 janvier 1905 fut accueilli par les socialistes français comme un événement capital qui marquait la fin programmée du tsarisme. Ce fut annoncé d'emblée et directement par le titre de l'éditorial de Jaurès dans L'Humanité du 23 janvier 1905 : « La mort du tsarisme ». Lucien Herr, russophone, ancien ami de Lavrov et responsable de la politique internationale dans L'Humanité de 1904-1905, écrivait tout aussi nettement : « quoiqu'il arrive - la révolution russe a cause gagnée ». Dès décembre 1904 au demeurant, malgré le coût élevé de la décision, le quotidien socialiste avait décidé d'être représenté à Saint-Pétersbourg par un correspondant permanent, Étienne Avenard, mesure aussi exceptionnelle que significative de l'attente de grands événements. Avenard resta en fonction jusqu'en juin 1905. Bien plus qu'à des considérations pessimistes sur les suites du mouvement, son retrait s'expliqua par les très graves difficultés financières que connut alors le journal de Jaurès et qui le contraignit aux plus sévères restrictions ${ }^{10}$. Toute l'année 1905, la presse socialiste, quelle que fût son orientation propre [Parti socialiste français (PSF) de Jaurès, Briand et Viviani, Parti socialiste de France (PSDF) de Guesde et Vaillant, Parti ouvrier socialiste révolutionnaire (POSR) d'Allemane, ou autres, avant le congrès de fusion d'avril 1905], publiait de nombreux articles qui informaient militants et sympathisants sur la situation en Russie et leur laissaient espérer une issue favorable. Ce ne fut que progressivement, au cours de l'année 1906, que l'attention se relâcha et que la défaite au moins provisoire de la révolution sembla admise. Ce fut sans doute le mois de juillet 1906, avec la dissolution facilement imposée de la Première Douma puis la formation du gouvernement Stolypin, qui signifia effectivement pour les socialistes français le vrai coup d'arrêt de la révolution. Auparavant, les divers temps forts du mouvement avaient été le plus souvent présentés comme marquant le dernier assaut. Juillet 1905 : la révolution s'étendait en province, la révolte du Potemkine annonçait la défection de l'armée ; automne 1905 : la vague de grèves qui précéda la manifestation du 17/30 octobre montrait l'isolement croissant d'un régime affaibli que décembre 1905 semblait à nouveau condamner avec l'insurrection de Moscou, saluée après son échec par une nouvelle vague de meetings en janvier 1906, organisés à l'occasion du premier anniversaire du Dimanche rouge. Néanmoins, jusqu'à l'été 1906 (soit en un peu plus de deux ans) Jaurès avait consacré au moins quarantecinq éditoriaux aux questions russes dans L'Humanité ${ }^{11}$.

Affaire de militants, pourrait-on objecter? Sans doute, mais pas seulement. Les socialistes s'efforcèrent d'élargir la solidarité. L'Humanité en appelait «aux civilisés $»^{12}$, repoussant le tsarisme et ses défenseurs dans une barbarie au-delà des

\section{L'Humanité, 26 novembre 1904.}

10. En mai 1906, le poste de correspondant fut confié à un militant russe, Nikolaj Kudrin.

11. Il en écrivit 10 en 1904, 28 en 1905, 7 en 1906, auxquels il faudrait ajouter ceux des autres responsables du journal - Viviani, Pressensé, et surtout Herr - , ainsi que les nombreux articles de Longuet ou Rémy, les dépêches d'Avenard....

12. L'Humanité, 24 janvier 1905. 
champs du débat politique ordinaire. De nombreux intellectuels, habitués au moins depuis les luttes dreyfusardes à prendre position, étaient sollicités : des écrivains comme Anatole France, Mirbeau, Descaves, les frères Margueritte, mais aussi Alfred Capus ou Marcel Prévost, voire Rodin, Maillol et Monet pour la libération de Gor'kij, brièvement emprisonné, des historiens avec Seignobos et Pirenne, des savants tels que Marcelin Berthelot ou Painlevé... La Ligue des droits de l'Homme était alors présidée par Francis de Pressensé, longtemps éditorialiste de politique étrangère au Temps (responsable du «Bulletin de l'étranger» de 1888 à 1905), député socialiste de Lyon et défenseur attitré de la cause arménienne, animateur de Pro Armenia... et coresponsable avec Lucien Herr de la politique internationale à L'Humanité, puis seul en lice à partir du mois d'août ${ }^{13}$. Sous les auspices de ce spécialiste reconnu de politique internationale, qui avait du reste soutenu activement l'alliance franco-russe et tenu autrefois des propos flatteurs sur la solidité du régime tsariste, se constitua en février 1905 une Association des amis du peuple russe et des peuples annexés présidée par Anatole France, alors au faîte de sa gloire et membre de l'Académie française. Cette association s'appuyait largement sur la sociabilité du salon d'Aline Ménard-Dorian, une égérie du régime républicain, nettement engagée à gauche. Elle regroupa des intellectuels, des socialistes et des radicaux de gauche, du moins pour ces derniers ceux alors délivrés de responsabilités gouvernementales comme Buisson, Pelletan ou le jeune Herriot ${ }^{14}$. Indice à ne pas négliger : les campagnes de souscription pour les révolutionnaires russes furent nombreuses (notamment celles de L'Humanité, du Socialiste, du Comité universitaire de protestation, de l'Osvoboždenie, le journal de Struve, des anarchistes Temps nouveaux...), alimentées par plusieurs dizaines de milliers de personnes et permirent de mobiliser des sommes relativement importantes (34 000 francs en tout) ${ }^{15}$.

\section{Les socialistes français ont-ils été unanimes devant la révolution de 1905 ? Leurs analyses différentes sont-elles fonction d'appartenances idéologiques diverses ?}

L'opposition au tsarisme était certes un souvenir commun à l'opinion démocratique et libérale, au moins depuis Hugo et Michelet. Seulement, au centre et à droite, et souvent à gauche aussi, la tradition démocratique et libérale restait seconde par rapport aux exigences nationales, donc cette opposition de principe était recouverte pour des raisons stratégiques. Elle ne disparaissait pas tout à fait pour autant. Les socialistes, à l'écart de toutes responsabilités gouvernementales directes depuis 1902, n'avaient pas de difficultés à clamer leur souhait, leur attente, leur conviction

13. Cf. Rémi Fabre, Francis de Pressensé et la défense des Droits de l'Homme. Un intellectuel au combat, Presses universitaires de Rennes, 2004.

14. L'Humanité, 3 et 26 février 1905.

15. Cf. Gilles Candar, «Les socialistes français et la révolution russe de 1905 », Jean Jaurès, bulletin de la Société d'études jaurésiennes, 131, janvier-mars 1994. 
de la chute prochaine du tsarisme. Ce consensus primordial n'empêchait pas des divergences sur les solutions de remplacement. On sait que pour les mencheviks russes, la révolution devait déboucher sur une étape démocratique et libérale. L'histoire ne faisant pas de saut, le prolétariat devait se développer et mûrir avant de mener à bien sa propre émancipation révolutionnaire qui permettrait d'établir le socialisme. La Social-démocratie devait donc avant tout appuyer l'opposition libérale bourgeoise. Les bolcheviks pensaient possible un prompt basculement de la révolution démocratique vers une révolution socialiste, en se fondant sur une alliance privilégiée avec la paysannerie ${ }^{16}$. Trockij, quoique situé dans la mouvance menchevik, se singularisait en allant encore plus loin que Lenin dans l'espérance d'une solution révolutionnaire qui pourrait dépasser l'étape de la révolution démocratique (1905, Bilan et perspectives).

Ces clivages russes, grossièrement rappelés ici, ne furent ni absolument étrangers, ni semblables à ceux qui se manifestèrent en France. La situation se complexifia d'autant plus que les lignes de démarcation nationales bougèrent et évoluèrent substantiellement. Au départ, l'opposition entre les deux principaux courants du socialisme français (PSF et PSDF) semblait se vérifier sans surprise, sur ce sujet comme sur bien d'autres. La possibilité d'une révolution prolétarienne était considérée comme possible et bien sûr souhaitable par de nombreux responsables et théoriciens socialistes, avant même que ne se déploie le mouvement révolutionnaire. C'était le cas de l'helléniste Bracke, compagnon de Guesde et défenseur attitré du marxisme, qui reprenait une analyse de Franz Mehring parue dans la Leipziger Volkszeitung (Le Socialiste, 21 février 1904). Paul-Louis, un jeune essayiste plein d'avenir, vaillantiste donc uni aux guesdistes dans le PSDF, mais avec des bases théoriques différentes, pensait de même. Dubreuilh, le lieutenant de Vaillant, parlait explicitement de « brûler les étapes ». En revanche, à L'Humanité, Jaurès et Lucien Herr faisaient entendre un autre langage. Ils souhaitaient un «commencement de régime constitutionnel», donc une révolution libérale qui pourrait parcourir plusieurs étapes (L'Humanité, 22 janvier 1905). C'est ce que pensaient aussi les allemanistes du POSR, ces socialistes anticléricaux et antimilitaristes, plutôt jauressistes que guesdistes, mais pas du tout favorables à des alliances avec les radicaux et à des compromis parlementaires ou bourgeois. Jaurès se distinguait aussi des guesdistes par une approche plus compréhensive de la question des emprunts russes. Il prévenait simplement les souscripteurs qu'ils avaient plus à craindre d'une perpétuation du tsarisme que de sa chute, garante d'un développement plus harmonieux ${ }^{17}$. Bracke, lui, se délectait à l'avance de leur déconvenue future dans un article au titre sans ambages : «Gare à vos écus ${ }^{18}$.

16. Le texte canonique de Lenin sur le sujet fut Deux tactiques de la Social-démocratie dans la révolution démocratique, rédigé à Genève en juin-juillet 1905, et vite publié par divers centres d'impression militants en Russie.

17. L'Humanité, 25 janvier 1905.

18. Le Socialiste, 5 février 1905. 
Cette différence s'accompagnait d'une connaissance assez poussée, chez les responsables du moins, des diverses analyses débattues en Russie. Ainsi, Le Socialiste, organe de référence lu par les cadres du parti, secrétaires fédéraux et secrétaires de section notamment (diffusion revendiquée de 4000 exemplaires), publia le 25 juin 1905 les principaux documents du III e congrès du POSDR, donc de la fraction bolchevik. Le 23 novembre 1905, Longuet, pourtant très lié personnellement aux mencheviks dont il partageait l'orientation stratégique et les analyses, publia une interview de Ladov qui expliqua plus clairement et succinctement les mêmes thèses aux lecteurs de L'Humanité. Une première approche pourrait conduire à faire correspondre le point de vue du PSDF (Guesde, Vaillant) à celui des bolcheviks, tandis que le PSF (Jaurès, Briand, Viviani) et le POSR (Allemane) seraient proches des mencheviks. Bref, un pôle plus « réformiste » et un autre davantage « révolutionnaire », ce qui ne manquerait pas de réjouir les tenants de l'historiographie des « deux cultures » au sein de la gauche socialiste française et internationale ${ }^{19}$.

Mais tout changea et se compliqua. Ce clivage, celui de 1904 et des premières semaines de la révolution, ne se maintint pas. Il se modifia au fur et à mesure que le mouvement révolutionnaire se confirma en Russie, et aussi en fonction des évolutions de la situation intérieure de la politique française, notamment chez les socialistes. La fin du Bloc des gauches et l'unification des socialistes jouèrent pour une expression plus ardente des positions de Jaurès et de ses amis entrés à la SFIO. En revanche, Briand, Viviani, qui préféraient maintenir l'alliance de la gauche républicaine et qui devinrent vite ministres (tous deux au cours de l'année 1906, au printemps pour le premier, à l'automne pour le second) allaient désormais être solidaires de la diplomatie française, du moins de celle mise en œuvre par les radicaux, à partir de mars 1906 (Léon Bourgeois, puis Stephen Pichon dans les cabinets Sarrien et Clemenceau). Auparavant, pendant l'année de transition, de réflexion et de détermination que fut pour eux 1905, ils avaient plutôt fait profil bas sur le sujet.

En revanche, Jaurès et ses amis de L'Humanité élevèrent dès le printemps 1905 le niveau de leurs exigences : le nouveau régime russe devait apporter des « garanties à la classe ouvrière et à la paysannerie » : propriété foncière « réelle » pour celle-ci, à l'instar du modèle français, journée de huit heures et autres réformes pour celle-là. Sans doute, ces réformes pouvaient toujours entrer dans le cadre d'une révolution démocratique. Cependant, des résultats plus ambitieux parurent vite envisageables : « le tsarisme est à bout [...] c'est un gouvernement populaire et national, c'est un gouvernement républicain qui se substituera au tsar », proclama dès lors Jaurès ${ }^{20}$. Au congrès de Chalon, en novembre 1905, ce furent bien « la destruction du régime capitaliste et la révolution sociale » qui furent envisagées, objectifs qui laissaient loin derrière ceux proclamés dans le manifeste des organisations socialistes et syndicales réunies à Tivoli à la fin janvier 1905. Paroles de congrès, qui n'engageaient pas davantage ? Le même congrès fut bien l'occasion d'une brève passe d'armes entre

19. Tel par exemple le regretté Jacques Kergoat, Histoire du Parti Socialiste, P. : La Découverte, « Repères », 1997.

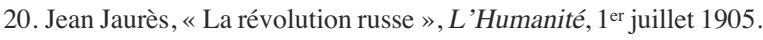


Lafargue et les jauressistes sur l'attitude à adopter face aux souscripteurs d'emprunts russes qui rappelaient les fortes nuances de l'année précédente. Il serait néanmoins fallacieux de ne pas prendre la mesure de l'évolution de Jaurès et de nombre de socialistes. Ainsi, les rapports entre Jaurès, L'Humanité et les démocrates russes libéraux et constitutionnels s'étaient nettement dégradés depuis le printemps 1905. Tout s'était joué autour de l'échange de lettres entre Struve et Jaurès publiées par L'Humanité les 8 et 10 juin 1905. Malgré l'aménité encore manifestée de part et d'autre, les raisons du désaccord de fond y étaient exprimées. Jaurès reprochait en somme à Struve d'ignorer le mouvement révolutionnaire. Sa critique ne laisse pas de faire penser au mot fameux de Robespierre : «vous vouliez une Révolution sans révolution ». En décembre 1905, la rupture fut nette lorsque le quotidien français prit position en faveur des socialistes russes et du soulèvement populaire contre les tentatives de compromis des cadets.

L'entraînement révolutionnaire emportait Jaurès, alors qu'au même moment, pourrait-on s'amuser à observer, Trockij élaborait sa théorie de la « révolution permanente », et le faisait renouer avec les aspects les plus ardents du messianisme révolutionnaire des années 1890 : « alors, - s'écria-t-il dans le meeting de l'ElyséeMontmartre, en décembre 1905, - toutes les chaînes seront brisées, toutes les geôles seront ouvertes, tous les bagnes sociaux vidés ». Jaurès évoluait, à la fois parce qu'il cherchait à prendre en compte les nouvelles conditions de l'époque, et parce que le souvenir de la Révolution française pesait tout particulièrement sur lui. Il venait au reste d'achever l' Histoire socialiste de la Révolution française. Il continuait à consacrer à ce sujet une bonne part de ses rares loisirs, animant une commission de publication des documents économiques et sociaux liés à la Révolution. Cette culture révolutionnaire était assez répandue au sein des élites républicaines. Elle avait entre autres pour conséquence d'avoir rendue familière aux socialistes français l'idée d'une révolution qui allât plus loin que ses buts initiaux. Qu'un nouveau «1789» puisse se prolonger en « 1792 » ou en « 1793-1794 » en Russie aussi ne les surprenait pas et ne pouvait leur déplaire, puisqu'aussi bien cela vérifiait en passant l'exemplarité de l'histoire nationale française... Il était fréquent de voir assimilés les divers protagonistes de la scène politique russe aux acteurs de la Révolution française : Nicolas II était Louis XVI, Witte : Necker, le prince Trubeckoj : La Fayette, Gapone : Mirabeau... Cela correspondait sans difficulté avec le volontarisme politique de la culture française, héritier de cette même tradition révolutionnaire.

De même, l'ancien communard Vaillant, quelle que fût sa contribution propre à la diffusion des thèmes marxistes dans le mouvement socialiste ${ }^{21}$, ne pouvait pas oublier pour autant son ascendance blanquiste et sa conviction que des minorités résolues pouvaient forcer le destin, même si les conditions d'évolution de la base économique et sociale n'étaient pas arrivées à pleine maturité. Le mouvement ouvrier français était habitué à affirmer une volonté d'action plus résolue face à une social-démocratie allemande, admirée ou redoutée, impressionnante mais aussi

21. Cf. Jolyon Howorth, Édouard Vaillant. La Création de l'unité socialiste en France, EDI/ Syros, 1982. 
rivale. Jaurès avait dénoncé son « impuissance » politique fondamentale au congrès international d'Amsterdam, Hervé commençait à populariser les thèmes de la grève générale, déjà très présents dans le monde syndical depuis plusieurs années et les campagnes de Pelloutier et de Briand en sa faveur. En fait, la vision étapiste des mencheviks russes convenait davantage à l'orthodoxie marxiste de la social-démocratie allemande. En France, elle était reprise par Jean Longuet, un petit-fils de Karl Marx, rédacteur de politique extérieure à L'Humanité, ami du Dachnak arménien (il était avec eux la cheville ouvrière du comité Pro Armenia et de son bulletin ${ }^{22}$ ), lié aussi à de nombreux Russes dont Steklov. Mais il était bien le seul ! En France, les rares responsables qui s'intéressaient à la politique extérieure avaient de toute façon une culture politique façonnée par une tradition révolutionnaire marquée par une forte autonomie du politique par rapport à la «base » économique et sociale.

Ajoutons que même les guesdistes, les plus proches des marxistes allemands, et en principe les plus déférents, laissaient parfois s'exprimer un certain agacement devant l'excessive prudence de leurs amis. Aussi bien sur le plan doctrinal, par exemple à propos de la question agraire (programmes de Marseille et de Nantes) que sur les formes d'action du mouvement socialiste, les audaces rhétoriques ne leur étaient pas absolument étrangères. Paul Lafargue ne fut pas toujours si mécontent de passer parfois pour «l'oncle » des « révolutionnaires » affichés du courant hervéiste ${ }^{23}$. Bien avant le déclenchement du processus révolutionnaire, il avait luimême établi un parallèle très précis entre ce qui s'était passé en France avec la Révolution française et les événements souvent identiques au détail près qu'il prévoyait pour la Russie 24 . La question de la violence des méthodes utilisées pouvait-elle diviser les socialistes ? Si Viviani montra bien qu'il comprenait davantage la politique de Struve et des constitutionnels démocrates que celle des révolutionnaires, le différend restait alors feutré et certainement peu apparent aux yeux de la masse des militants et des sympathisants ${ }^{25}$. Les socialistes et même l'ensemble de la gauche républicaine restaient assez proches de leurs origines révolutionnaires pour ne pas s'effaroucher de l'emploi de méthodes qui pouvaient paraître obsolètes dans une démocratie, mais légitimes et nécessaires justement en régime autocratique comme elles l'avaient été pour abattre l'Ancien Régime. Ainsi, les attentats individuels contre des dignitaires du régime étaient justifiés et approuvés. Celui visant Plehwe en juillet 1904 avait été unanimement fêté. Le président de la Ligue des droits de l'Homme, Francis de Pressensé, encore employé au Temps et bon connaisseur de la cour impériale, écrivait sans ambages après l'assassinat du grand-

22. Côté arménien, voir Anaïde Ter Minassian, La Question arménienne, Roquevaire: Parenthèses », 1983. Du côté socialiste français, cf. Rémi Fabre, Francis de Pressensé, op. cit. et Gilles Candar, Jean Longuet (1876-1938), Presses universitaires de Rennes, 2007.

23. Sur Paul Lafargue et la révolution russe de 1905, cf. Jacques Girault, « Les conséquences de la révolution de 1905 sur le socialisme français : l'exemple de Paul Lafargue », in FrançoisXavier Coquin et Céline Gervais-Francelle, éds., 1905. La première révolution russe, P.: Publications de la Sorbonne, 1986.

24. Paul Lafargue, « La fin du tsarisme », Le Socialiste, 28 février 1904.

25. René Viviani, « Dupeur dupé », L'Humanité, 24 août 1905. 
duc Serge : «il n'est plus qu'une seule ressource : la force $»^{26}$. Le sage et docte bibliothécaire de l'École normale supérieure, Lucien Herr, parlait pour sa part de 《justes représailles » 27 . Il précisait : « la révolution frappe bien et vise juste » et présentait le défunt en ces termes peu flatteurs : « il avait à la fois toute la bassesse du voleur et du coquin le plus vil, et toute la cruauté hautaine et audacieuse du bourreau [...] il étalait avec un égal cynisme ses vices de détraqué et l'insolence de ses rapines $[\ldots]$ brigand à l'âme sinistre et hideuse ».

\section{La révolution de 1905 a-t-elle influé sur la politique française ? Sur les équilibres internes au socialisme et à la gauche française?}

Directement, sans doute pas. Il faut tenir compte d'abord du fait que le socialisme français ne pensait pas aisément avoir des leçons à recevoir de l'étranger. La fierté nationale était constamment affirmée. Même le courant le plus internationaliste dans son discours, celui des marxistes Guesde ou Lafargue, était attentif à marquer son caractère patriotique. Les Français avaient de la sympathie pour les révolutionnaires russes, ils pouvaient admirer leur courage, depuis les images assez littéraires des jeunes femmes du socialisme révolutionnaire capables de sacrifier leurs vies pour un idéal jusqu'aux manifestants qui affrontaient les cosaques dans les grèves ou les manifestations. Mais ils gardaient le sentiment d'appartenir à un pays plus avancé, et l'image folklorique de révolutionnaires russes aimant trop la discussion et prompts à se diviser était bien ancrée dans les représentations socialistes. Ceci apparaît du moins dans la presse ainsi que dans quelques témoignages parfois ultérieurs sur les Russes (voir par exemple les carnets de Lucien Roland ${ }^{28}$ ). Le rédacteur de politique internationale à L'Humanité, Jean Longuet, se servit ainsi de la révolution russe non pour mener à bien une réflexion théorique, qui aurait discuté des «thèses » avancées par les uns et par les autres, mais pour publier un ouvrage de reportage, Terroristes et policiers, à propos de «l'affaire Azeff», qui ne répugnait pas à sacrifier au « pittoresque », chez Juven, un éditeur populaire spécialisé dans les livres pour la jeunesse. Son livre fut d'ailleurs traduit en allemand et suédois. Les socialistes français ne cessaient d'en appeler à l'unité de leurs homologues russes. Du moins dès que leur propre unité fut réalisée en France, ils purent se donner en exemple aux Russes. Ce parti pris ou cette incompréhension expliquèrent la popularité de personnalités qui semblaient échapper aux querelles de «sectes » tel pendant un moment le pope Gapone que Longuet défendit même contre Plekhanov dans L'Humanité.

Il serait excessif de s'imaginer, comme semblait le croire Manfred, un mouvement socialiste français se transformant parce que 1905 aurait fait luire une première lueur à l'est. L'histoire de l'unité socialiste en France fut longue et

26. L'Humanité, 19 février 1905.

27. Lucien Herr, « Les justes représailles », L'Humanité, 19 février 1905.

28. Les carnets de Lucien Roland sont conservés à l'Institut français d'histoire sociale (Archives nationales) dans le fonds Maurice Dommanget (14 AS 280). 
complexe, elle intervint au terme d'un processus compliqué, dans lequel les pressions de l'Internationale socialiste jouèrent un rôle, mais ses causes fondamentales n'en restèrent pas moins liées à la situation intérieure française, du côté des divers partis socialistes comme de leurs partenaires ou adversaires. Que la révolution russe ait néanmoins permis de mettre sur pied de belles manifestations d'unité, que la sympathie dans le peuple militant et sympathisant ait été réelle, sans doute. Un journal avisé comme Le Temps (auquel collaborait peut-être encore Francis de Pressensé) le remarqua: «L'affaire russe vient à point nommé pour exhiber M. Allemane, M. Guesde, et M. Jaurès sur la même estrade. On s'accorde donc sur l'affaire russe, cette agitation durera peu de temps, mais encore assez pour faire accepter aux électeurs le compromis socialiste ${ }^{29} \gg$. Solidarité sans doute plus agissante et ressentie à Paris et dans les grands centres urbains, où elle s'insérait aisément dans la mémoire communarde : Vaillant présida le meeting commun de Tivoli (30 janvier 1905), celui du 11 décembre de la même année, à l'Elysée-Montmartre le fut par Jean Allemane, l'autre communard emblématique du socialisme français, à nouveau sollicité pour ce qui constitua le dernier grand meeting de solidarité, tenu au Cirque d'hiver le 7 juillet $1906^{30}$. Le troisième homme au reste du souvenir communard chez les socialistes, Camélinat, «l'orgueil du pays », avait dressé dès le 22 janvier 1905 un parallèle fécond et évocateur entre les deux mouvements lors des obsèques de Louise Michel. D'ailleurs, si à Paris, le meeting commun pour la révolution russe scella l'unité socialiste parmi les militants, en province, si des réunions furent aussi organisées avec des succès divers ${ }^{31}$, d'autres occasions furent souvent jugées plus propices. Dans le Midi, cela fut à Béziers et à Maraussan autour du thème fédérateur de la défense de la vigne, avec les premières difficultés de mévente et de surplus, qui avait amené la réalisation de coopératives...

Sinon, pour l'essentiel, les socialistes français étaient davantage enclins à penser que les Russes devaient plutôt chercher à suivre leur exemple que l'inverse. L'heure pour les Russes devait être au rattrapage. Qu'ils s'inspirassent de la Révolution française de 1792-1794, des « rouges » de 1849-1851, ou des Fédérés de la Commune de 1871 était compréhensible et sympathique. Une attitude inverse de la part des militants du mouvement ouvrier français, héritier d'une glorieuse tradition révolutionnaire, restait peu concevable. Davantage encore que la question de l'unité organisationnelle, celle de la participation aux élections révélait au demeurant l'ampleur des différences. Le boycottage de la Douma fut très mal compris de militants pour qui la participation aux élections, héritage d'une culture républicaine, avait servi d'élément discriminant pour se distinguer de l'anarchie. Si cette politique se comprit pour la première assemblée projetée, dite Douma Boulygin, non élue au suffrage universel et

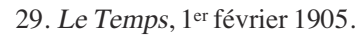

30. Cf. Madeleine Rebérioux, «Le mur des fédérés. Rouge, "sang craché” », in Pierre Nora, éd., Les Lieux de mémoire, I. La République, P. : Gallimard, «Bibliothèque illustrée des histoires », 1984.

31. 21 meetings les 18 et 19 février. Affluence record semble-t-il à Lille, avec Jaurès, Sembat et Delory, mais outre la notoriété des orateurs, Lille est une ville clef du processus unitaire, déception à Decazeville avec Camélinat. 
sans pouvoir délibératif, malgré déjà les réserves de Viviani ${ }^{32}$, les dirigeants socialistes ne respectèrent que des précautions formelles pour ne pas dire davantage qu'ils n'approuvaient pas ni comprenaient la poursuite de cette politique avec la Première Douma effectivement élue, réunie en mai 1906. Bracke, à sa manière d'apparente franchise bougonne, le formula assez clairement ${ }^{33}$. Les sociaux-démocrates russes abandonnèrent d'ailleurs cette position, participèrent à la Deuxième Douma (marsjuin 1907) et Lenin devait plus tard qualifier d'erreur gauchiste le boycottage initial de $1906^{34}$. Au demeurant, il est assez remarquable que si dans la presse socialiste, les ordres du jour de réunions manifestaient une grande attention et beaucoup de sympathie pour le mouvement révolutionnaire russe, cela ne se retrouvait guère dans les discussions doctrinales, les articles ou les études de revues. Le Mouvement socialiste de Lagardelle, pourtant fortement engagé dans l'approfondissement des questions de grève révolutionnaire et d'insurrection, ne publia aucun article sur la Russie après avril 1905. Encore s'agissait-il dans cette dernière occasion d'un texte informatif [sur le Bund] écrit par un militant étranger. Cette abstention se retrouva dans les autres revues de la mouvance socialiste ${ }^{35}$, à la seule exception de La Vie socialiste. Il est vrai que La Vie socialiste était codirigée par Francis de Pressensé et Jean Longuet, deux spécialistes de politique internationale et tous deux intéressés au premier chef par les questions russes. De plus, cette publication «jauréssienne de gauche» ne pouvait que se passionner pour un mouvement à la fois démocratique, révolutionnaire et prolétarien qui accompagnait le « tournant à gauche » de Jaurès au printemps 1905. Enfin, et de toute façon, la réception de La Vie socialiste ne peut être surestimée puisque la revue fut arrêtée dès août $1905^{36}$. Les rares analyses rencontrées se caractérisèrent par la prise de distance entre la situation française et celle de la Russie. Pour une fois, le guesdiste orthodoxe Charles Bonnier et Georges Sorel se rencontrèrent pour marquer toutes les différences entre la grève générale telle qu'entendue en France et celle pratiquée en Russie ${ }^{37}$.

Pour autant, il n'est pas absurde de s'interroger sur une influence indirecte. La révolution russe aida les socialistes français à persévérer dans la voie qu'ils avaient commencé à emprunter : critique accentuée de l'alliance franco-russe ${ }^{38}$ et dévelop-

32. Art. cit., L'Humanité, 24 août 1905. Jaurès lui-même avait paru enclin à la participation, citant la formule de Tocqueville : « la démocratie est comme la tombe, elle prend toujours et ne rend jamais », « Vers l'abîme », L'Humanité, 27 août 1905.

33. Bracke, « La Douma », L'Humanité, 18 mai 1906.

34. Cf. V. I. Lénine, La Maladie infantile du communisme, le «gauchisme », 1920, rééd. P. : Éd. sociales, 1969, p. 21-22.

35. La Revue socialiste dirigée alors par Fournière reste visiblement en dehors de ce nouveau champ.

36. Cf. Gilles Candar, «La Vie socialiste, 1904-1905. Une revue jauressiste ? , Cahiers Jean Jaurès, 146, octobre-décembre 1997, p. 11-16.

37. Charles Bonnier, «La Grève générale en Russie », Le Socialiste, 18 novembre 1905 et Georges Sorel, Réflexions sur la violence, P. Rivière, 1908, rééd. Michel Prat, P. : Seuil, 1990.

38. Le plus net fut encore Jaurès, évoquant le « scandale », « la complicité atroce » dans « La Fin d'une alliance », L'Humanité, 3 février 1905. 
pement du thème de la grève générale. Le mot juste semble trouvé par Madeleine Rebérioux qui retient à cet égard « l'atmosphère d'exaltation »39 à laquelle participaient, peu ou prou, selon les moments et les occasions, les événements russes. Mais les socialistes français firent aussi et surtout le constat que la révolution russe finalement échouait. C'était un nouveau signe de la retombée des espérances révolutionnaires et du messianisme révolutionnaire. Avec la croissance économique, l'heure était plutôt à une évolution longue, subtile et complexe. Il fallait conclure des alliances nécessaires. La priorité était d'établir un régime de démocratie et de liberté. L'État disposait d'une capacité propre de détermination. Ses réflexions furent au cœur de l'intervention de Jaurès au congrès de Toulouse (octobre 1908) qui chercha explicitement à tirer les leçons de l'échec de la révolution russe et devint un classique de la doctrine socialiste française. Dans le même ordre d'idées, L'Armée nouvelle (1911), qui marqua l'aboutissement provisoire de la réflexion jaurésienne sur l'État, prenait en compte les événements de la Russie en révolution. La Russie était-elle cependant vraiment analysée alors pour elle-même, ou plutôt utilisée pour donner matière à des argumentations qui lui restaient étrangères ? La révolution de 1905 intéressait d'autant plus le socialisme français qu'elle était intégrée à l'histoire nationale. L'universalisme français fonctionnait plutôt bien. Il permettait, voire favorisait intérêt et ouverture sur le monde, mais au prix d'une francisation des problèmes. L'ambiguiité du message universel de la Révolution française qui peut se retrouver chez Hugo (les États-Unis d'Europe conçus comme une extension de la République française) ou chez De Gaulle (« il est un pacte entre la grandeur de la France et la liberté des peuples ») existait sans doute aussi chez Jaurès, Guesde ou Vaillant. De toute façon, la France restait la mesure de toutes choses.

En somme, si le constat d'un intérêt massif des socialistes français pour la révolution russe ne peut être mis en doute, s'il appartient au domaine des faits facilement vérifiables et irrévocables, la discussion peut se poursuivre sur la part d'influence de ces événements au sein du socialisme français. Il serait aussi peu crédible de reprendre une historiographie soviétique, qui répondait au demeurant à des demandes sociales et politiques particulières, que de s'en tenir à une histoire parallèle trop linéaire, mais l'analyse de la révolution russe agissant comme révélateur de stratégies, de comportements et de cultures politiques diverses au sein du mouvement socialiste et la compréhension de la manière dont elle fut utilisée, pensée et intégrée à la construction de la doctrine socialiste, au premier chef par Jaurès, pourraient être à nouveau très utilement travaillées.

\author{
Société d'études jaurésiennes \\ WWW.jaures.info \\ gilles.candar@wanadoo.fr
}

39. Madeleine Rebérioux, La République radicale ?, P. : Seuil / « Points Histoire », 1975, p. 104 . 